Enfermagem Brasil 2018;17(4):308-17

\title{
ARTIGO ORIGINAL \\ Estratégias para melhoria da qualidade dos materiais hospitalares licitados em pregão eletrônico
}

Fábio Vilas Gonçalves Filho, M.Sc. ${ }^{*}$, Luiz Eduardo da Motta Ferreira**, Carmem Fernandes Alves, M.Sc. ${ }^{* *}$, Liane Gack Ghelman, D.Sc. ${ }^{* \star *}$, Ediclea Mascarenhas Fernandes, D.Sc. ${ }^{* * * *}$, Luciana Krauss Rezende, D.Sc. ${ }^{* * * * *}$, Nébia Maria Almeida de Figueiredo, D.Sc. ${ }^{* * \star * * *}$, Wiliam César Alves Machado, D.Sc. ${ }^{* * * * * * *}$

*Advogado, Programa de Pós-Graduação em Saúde e Tecnologia no Espaço Hospitalar PPGSTEH, Universidade Federal do Estado do Rio de Janeiro (UNIRIO), "*Médico, Professor Adjunto, Escola de Medicina e Cirurgia, UNIRIO, ${ }^{* * * E n f e r m e i r a, ~ M e s t r e ~ e m ~ S a u ́ d e ~ e ~ T e c n o l o g i a ~}$ no Espaço Hospitalar - PPGSTEH, UNIRIO, Hospital Universitário Gafré e Guinle - HUGG, ${ }^{* * \star * E n f e r m e i r a, ~ P r o f e s s o r ~ A d j u n t o ~ n o ~ D e p a r t a m e n t o ~ d e ~ E n f e r m a g e m ~ d e ~ S a u ́ d e ~ P u ́ b l i c a, ~ E s c o l a ~}$ de Enfermagem Anna Nery (EEAN), Universidade Federal do Rio de Janeiro (UFRJ),

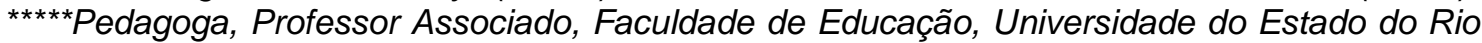
de Janeiro (UERJ), ${ }^{* * * * * *}$ Fisioterapeuta, Professor na Faculdade Univértix de Três Rios (UNIVÉRTIX), ${ }^{* * * * * * * E n f e r m e i r a, ~ P r o f e s s o r a ~ E m e ́ r i t a, ~ D e p a r t a m e n t o ~ d e ~ E n f e r m a g e m ~}$ Fundamental, Escola de Enfermagem Alfredo Pinto (EEAP), UNIRIO, ${ }^{* * * * * * * \star}$ Enfermeiro, Professor Adjunto, Departamento de Enfermagem Fundamental, Escola de Enfermagem Alfredo Pinto (EEAP), UNIRIO, Professor na Faculdade Univértix de Três Rios (UNIVÉRTIX)

Recebido em 15 de maio de 2018; aceito em 24 de agosto de 2018.

Endereço de correspondência: Prof. Dr. Wiliam César Alves Machado RN, MsN, Ph.D, Universidade Federal do Estado do Rio de Janeiro, Rua Silva Jardim, 5, 25805-160 Três Rios RJ, E-mail: wilmachado@uol.com.br; Fábio Vilas Gonçalves Filho: fabiounirio@hotmail.com; Luiz Eduardo da Motta Ferreira: luizmotta@predialnet.com.br; Carmem Fernandes Alves: carmem.hrm@gmail.com; Liane Gack Ghelman: Igghelman@gmail.com; Ediclea Mascarenhas Fernandes: professoraediclea.uerj@gmail.com; Luciana Krauss Rezende: lukrare@uol.com.br; Nébia Maria Almeida de Figueiredo: nebia43@gmail.com

Estudo extraído da dissertação de mestrado intitulada "Implantação da comissão de pré-qualificação de materiais hospitalares no pregão eletrônico de hospital universitário: pré-requisito para controle de qualidade". Apresentada ao Programa de Pós-Graduação em Saúde e Tecnologia no Espaço Hospitalar - PPGSTEH, Universidade Federal do Estado do Rio de Janeiro (UNIRIO)

\section{Resumo}

Objetivo: Propor estratégias para que o pregão eletrônico de compras de materiais para hospitais universitários constitua-se num processo focado na qualidade dos produtos adquiridos, repercutindo em segurança dos pacientes e equipes de saúde. Métodos: Estudo descritivo, abordagem qualitativa, realizado com 11 médicos e 18 enfermeiros, em 2015. Entrevistas analisadas à luz da análise temática de conteúdo. Resultados: Da análise dos dados emergiram as seguintes categorias temáticas: Materiais com mais problemas, intercorrências por falta de critérios de qualidade; Credibilidade sobre solicitação de amostras para o pregão; e propósitos da comissão de padronização de materiais hospitalares. Conclusão: Sugerida a implantação de comissão para pré-avaliação de amostras, como recomendação estratégica essencial para melhoria da qualidade dos materiais hospitalares. $O$ artigo não esgota o tema, mas evidencia a necessidade de novos estudos sobre compras através de pregões eletrônicos, para se buscar critérios de pré-avaliação das amostras de materiais terapêuticos utilizados em hospitais universitários.

Palavras-chave: serviço hospitalar de compras, administração de materiais no hospital, administração hospitalar, controle de qualidade; assistência à saúde.

\section{Abstract}

Strategies to improve the quality of hospital materials purchased through electronic bids Objective: To propose strategies for the electronic procurement of materials for university hospitals to be a process focused on the quality of the products purchased, with repercussions on the safety of patients and health care teams. Methods: Descriptive study, qualitative approach, carried out with 11 physicians and 18 nurses. Interviews analyzed in the light of 
thematic content analysis. Results: From the data analysis, the following thematic categories emerged: Materials with more problems, intercurrences due to lack of quality criteria; Credibility for requesting samples for electronic procurement; and purposes of the hospital materials standardization commission. Conclusion: A commission for pre-evaluation of samples was suggested as an essential strategic recommendation for improving the quality of hospital materials. The article does not exhaust the theme but shows the need for further studies on public contracting regarding electronic procurement in order to seek criteria in the preevaluation of material samples for university hospitals.

Key-words: hospital purchasing, hospital materials management, hospital administration, quality control, delivery of health care.

\section{Resumen}

\section{Estrategias para mejorar la calidad de los materiales hospitalarios licitados en una sesión electrónica}

Objetivo: Proponer estrategias para que la licitación electrónica de compras de materiales para hospitales universitarios constituya un proceso enfocado en la calidad de los productos adquiridos, con repercusión en seguridad del paciente y equipos de salud. Métodos: Estudio descriptivo, abordaje cualitativo, realizado con 11 médicos y 18 enfermeros en 2015. Entrevistas estudiadas mediante análisis de contenido temático. Resultados: Del análisis de datos surgieron las categorías: Materiales más problemáticos; Incidencias por inexistencia de criterios de calidad; Credibilidad sobre solicitud de muestras para la licitación; y Propósitos de la comisión de estandarización de materiales hospitalarios. Conclusión: Sugerida la implantación de comisión de preevaluación de muestras, como recomendación estratégica fundamental para mejorar la calidad de los materiales hospitalarios. El artículo no agota el tema, aunque evidencia la necesidad de nuevos estudios sobre compras por licitación electrónica para buscar criterios de preevaluación de muestras de materiales terapéuticos utilizados en hospitales universitarios.

Palabras-clave: departamento de compras en hospital, administración de materiales de hospital, administración hospitalaria, control de calidad, prestación de atención de salud.

\section{Introdução}

Utilizado para dinamizar os processos de compras de materiais hospitalares, o pregão eletrônico, quando não contempla critérios de avaliação de amostras, não se configura modalidade de licitação satisfatória, considerando a celeridade e redução de custos que 0 mesmo propicia para a administração pública, contudo, não prioriza elementos de qualidade, essenciais na aquisição desses materiais $[1,2]$.

Sabe-se que as compras e contratações públicas compreendem procedimentos com características e componentes de caráter comercial, envolvendo a negociação de propostas de preço e de caráter jurídico-legal, sujeitando-se a controles [1], à interposição de recursos e à aplicação de sanções administrativas [2].

Importante considerar no contexto deste estudo que o número de hospitais de ensino, no Brasil, em 2013, era de 134 instituições distribuídas em todo o território nacional, com 80 públicas, 47 filantrópicas, e sete instituições privadas [13]. Identificadas como instituições de ensino, pesquisa e extensão na área de saúde, nas quais os gestores dos hospitais universitários devem primar pela qualidade dos serviços oferecidos a sociedade, sobretudo, pelo controle da qualidade dos materiais adquiridos, de forma a garantir segurança para os pacientes e profissionais que os utilizam.

De acordo com estudo realizado no Hospital Universitário da Universidade de São Paulo, o sistema de gestão de materiais é um dos grandes determinantes do planejamento financeiro de uma instituição, ou seja, é nesta área que se observa um grande gasto da receita e onde o capital poderá ser consumido [4].

No Brasil, a Lei no 8.666 de junho de 1993, é a principal norteadora dos processos de compras públicas, a qual foi alterada, pela redação conferida ao art. 30 da mesma lei, pela Lei no 12.349 , de 15 de dezembro de 2010, que modificou radicalmente o quadro jurídico e operacional das licitações públicas no Brasil, obrigando todos os entes da Federação a promoverem licitações públicas sustentáveis $[2,5,6]$.

O objetivo da redução de custos e a agilização de rotinas e procedimentos nas compras e contratações têm sido uma prioridade na administração federal brasileira [4]. A introdução de novos instrumentos, proporcionados pelas tecnologias da informática, ao lado da 
revisão da legislação e das normas, já experimentou avanços significativos. Após a implantação do pregão eletrônico, foram abertas possibilidades inéditas de maior transparência, ampliação de oportunidades de participação e de competição e disseminação de mecanismos de controle gerenciais [1-3,7].

Para fins norteadores dos aspectos jurídico-administrativos das compras de materiais hospitalares, objeto deste estudo, buscou-se respaldo na Lei № 10.520/2002, que criou a modalidade pregão, e no Decreto no 5.450/2005, que regulamentou o pregão eletrônico [5]. É importante destacar que a modalidade pregão, embora não verse sobre a questão, a doutrina e a jurisprudência do Tribunal de Contas da União, permitem aos gestores incluir cláusula impositiva de exigência de amostra ou protótipos de licitante, provisoriamente, nos editais, viabilizando a testagem dos profissionais que as utilizarão.

A questão norteadora desta investigação é: Como proceder para que o pregão eletrônico de compras de materiais hospitalares seja focado na melhoria da qualidade dos produtos adquiridos e repercutam na segurança dos pacientes e equipes de saúde?

Isto posto, considerando a necessidade de aprimoramento dos processos de compras através do pregão eletrônico, conferindo melhorias na qualidade do cuidado e assistência de saúde prestados à clientela, o objetivo deste estudo foi propor estratégias para que o pregão eletrônico de compras de materiais em hospitais universitários constitua processo focado na qualidade dos produtos adquiridos, repercutindo na segurança dos pacientes e equipes de saúde.

\section{Material e métodos}

Trata-se de estudo descritivo, de abordagem qualitativa, realizado no período de setembro a dezembro de 2015, com gestores de unidades de internação do Hospital Universitário Gafreé e Guinle, da Universidade Federal do Estado do Rio de Janeiro (UNIRIO), unidade da rede federal de ensino, de grande porte, localizado na Cidade do Rio de Janeiro/RJ/Brasil, fundamentado nas diretrizes da Política Nacional de Compras Sustentáveis [8].

Participaram deste estudo 18 enfermeiros e 11 médicos, atuantes em cargos de gestão de unidades de internação de média e alta complexidade, tratamento intensivo, de internação clínica, cirúrgica e de tratamento intensivo, cujas funções envolvem solicitação, avaliação e utilização de materiais hospitalares. Todos devidamente convidados a participar do estudo, baseado no especificado no seu Termo de Consentimento Livre e Esclarecido.

\section{Critérios de inclusão}

Exercer função de gestor de unidade de internação do Hospital Universitário Gafreé e Guinle, ser responsável pelo processo de compras e solicitação de materiais hospitalares, utilizados nos procedimentos terapêuticos realizados com os clientes da unidade, concordar em participar e assinar o termo de consentimento livre e esclarecido.

$O$ desenvolvimento do estudo atendeu as normas nacionais e internacionais de ética em pesquisa envolvendo seres humanos e animais, conforme parecer CAAE $n^{\circ}$ 47784415.8.0000.5285, aprovado em 27/08/2015, emitido pelo CEP UNIRIO.

\section{Caracterização da amostra}

Participaram do estudo 16 gestoras do sexo feminino (55.17\%) e 13 gestores do sexo masculino (44.83\%). A idade dos participantes variou entre 31 e 70 anos, com média de 47,72 anos. O tempo de formados variou entre 8 e 40 anos, tendo média de 24,38 anos, enquanto 0 tempo de atuação no setor obteve média de 14,54 anos. Quanto ao vínculo de atuação profissional, constatou-se 24 (82.76\%) assistenciais e 5 (17.24\%) docente e assistencial

\section{Produção de dados}

Ocorreu no período de setembro a dezembro de 2015, através da técnica de pesquisa de entrevista individual, cujo instrumento foi roteiro semiestruturado, elaborado pelos pesquisadores. As entrevistas foram agendadas com antecedência, procurando conciliar os 
compromissos dos participantes e dos pesquisadores, sendo realizadas na instituição hospitalar, em ambiente exclusivo, como medida preventiva a eventuais interrupções.

As entrevistas foram gravadas em áudio e posteriormente transcritas para o Word. 0 roteiro das entrevistas buscou responder as seguintes questões:

1. Qual o material de procedimento que apresenta mais problema (no quesito qualidade) no setor onde você atua?

2. Já ocorreu alguma intercorrência no procedimento, por causa da qualidade de algum material?

3. Você acredita que a solicitação de amostras dos produtos hospitalares no Pregão Eletrônico pode contribuir para que não ocorram essas intercorrências?

4. A criação de uma comissão para avaliação de amostras no pregão eletrônico minimizaria problemas de qualidade dos materiais?

Método para análise dos dados produzidos

Utilizou-se a análise de conteúdo, mais precisamente a análise temática [9], considerando-se suas três etapas: pré-análise (leitura flutuante e formulação de hipóteses), exploração do material (codificação e classificação em categorias), tratamento dos resultados obtidos e interpretação (processo de reflexão). O texto bruto foi decodificado em categorias de análise temática sobre como os gestores compreendem a dinâmica dos processos de compras e licitações de materiais hospitalares através dos pregões eletrônicos, com ênfase no controle da qualidade e segurança dos pacientes e clientes internados na instituição.

A análise das categorias baseou-se nos princípios básicos da Lei 8.666/93, utilizando os benefícios das novas tecnologias da informação (TI), como forma de propor mecanismos de controle de qualidade dos materiais comprados através dos pregões eletrônicos do Hospital Universitário Gafreé e Guinle.

Buscando preservar o anonimato dos participantes nos recortes apresentados nas categorias, decidiu-se pelo uso do seguinte esquema: [Profissão e número de ordem], considerando as legendas ENF. (enfermeiro 1-18), MED. (médico 1-11).

\section{Resultados}

Os dados brutos foram organizados em unidades de registro a partir dos temas que compõe os quadros abaixo, como forma de organização para a definição das categorias do estudo.

Quadro I - Materiais que apresentam mais problemas, Hospital Universitário, Rio de Janeiro, 2015.

\begin{tabular}{|l|l|}
\hline Ordem & Unidades de registro \\
\hline $\mathbf{1}$ & Jelcos: (ENF1, ENF2, ENF3, ENF4, ENF8, ENF11, ENF16, MED10). \\
\hline $\mathbf{2}$ & Luvas: (ENF1, ENF5 ENF10,0, ENF12, ENF18). \\
\hline $\mathbf{3}$ & Equipo. (ENF10, ENF11, ENF13). \\
\hline $\mathbf{4}$ & Polifix: (ENF9, ENF10, ENF11). \\
\hline $\mathbf{5}$ & Catéter venoso: (ENF13, MED3). \\
\hline $\mathbf{6}$ & Agulhas: (ENF10, MED5). \\
\hline $\mathbf{7}$ & Nenhum: (ENF14, MED4, MED9). \\
\hline \multicolumn{2}{|l|}{ Fonte: Participantes deste estudo, dados das entrevistas. }
\end{tabular}


Quadro II - Intercorrências com materiais por falta de qualidade, Hospital Universitário, Rio de Janeiro, 2015.

\begin{tabular}{|c|c|}
\hline Ordem & Unidades de registro \\
\hline 1 & $\begin{array}{l}\text { [...] no caso do jelco que é o cateter intravenoso curto chegou a rasgar a pele do } \\
\text { paciente (ENF3). }\end{array}$ \\
\hline 2 & $\begin{array}{l}{[\ldots] \text { teve essa questão do jelco }[\ldots] \text { por ele ser de má qualidade você precisa }} \\
\text { puncionar mais vezes o bebê }[\ldots] \text { acaba causando mais estresse, tanto na criança } \\
\text { quanto na equipe }[\ldots . .] \text { você acaba causando vários hematomas na criança. [...] de } \\
\text { não conseguir, você extingue todos os acessos venosos por conta de um material } \\
\text { ruim [...] ter que chamar o cirurgião (ENF } 2 \text { ). }\end{array}$ \\
\hline 3 & $\begin{array}{l}\text { [...] você não sabe como é o cateter, você põe o cateter ai tem o mandril que é o } \\
\text { guia a gente tira o guia e deixa lá dentro só o flexível na hora de tirar o guia agarra } \\
\text { no flexível ai você perdeu o acesso venoso tem que tirar aquele e puncionar outra } \\
\text { veia. Porque o material não é de boa qualidade (ENF 4). }\end{array}$ \\
\hline 4 & $\begin{array}{l}\text { [...] principalmente do jelco, que rasga a pele da pessoa [...] a ponta do jelco que não } \\
\text { penetra, causando dor }[\ldots] \text { é muito ruim tanto para o profissional que está } \\
\text { executando como para a pessoa que está recebendo o cuidado. [...] pela qualidade } \\
\text { inferior do material (ENF 8). }\end{array}$ \\
\hline 5 & $\begin{array}{l}\text { [...] tivemos que interromper a transfusão e ela não pode ser completa por conta da } \\
\text { qualidade (MED 1). }\end{array}$ \\
\hline 6 & $\begin{array}{l}{[\ldots] \text { ocorreu da gente precisar trocar o material durante o procedimento porque tinha }} \\
\text { vazamento ou porque o cateter tinha uma dobra quebrada [...] isso causa uma } \\
\text { demora no procedimento [...] às vezes a gente faz o procedimento, no dia seguinte } \\
\text { ou pouco tempo depois tem que realizar um novo procedimento no doente e acaba } \\
\text { expondo o doente aos riscos inerentes ao procedimento (MED } 3 \text { ). }\end{array}$ \\
\hline 7 & $\begin{array}{l}\text { [...] tivemos que interromper cirurgias porque o cateter duplo já não conseguia } \\
\text { correr sobre o fio guia hidrofílico [...] a qualidade é um fator muito importante para o } \\
\text { sucesso do procedimento cirúrgico e para diminuir a internação (MED } 7 \text { ). }\end{array}$ \\
\hline
\end{tabular}

Quadro III - Eficácia da solicitação de amostra para o pregão, Hospital Universitário, Rio de Janeiro, 2015.

\begin{tabular}{|l|l|}
\hline Ordem & Unidades de registro \\
\hline 1 & $\begin{array}{l}\text { Por que muitas vezes vem amostra insuficiente, amostra demora e geralmente são } \\
\text { sempre materiais de baixa qualidade (ENF2). }\end{array}$ \\
\hline 2 & Já tive algumas solicitações de amostras e supriu a necessidade (ENF3). \\
\hline 3 & $\begin{array}{l}{[\ldots . .] \text { já trabalhei no Ministério da Saúde onde eu implantei este método e tínhamos }} \\
\text { uma comissão de compra de material e esse material quando vinha, as amostras } \\
\text { vinham para a gente [...] fazíamos a avaliação e estas respostas eram arquivadas no } \\
\text { setor, então no próximo pregão a gente já sabia quais os produtos que eram de } \\
\text { qualidade e aqueles que não satisfaziam (ENF5). }\end{array}$ \\
\hline 4 & $\begin{array}{l}\text { Não é eficaz porque na verdade o tempo que você tem para testar é muito curto e a } \\
\text { quantidade enviada é muito pequena de amostra (ENF10). }\end{array}$ \\
\hline 5 & $\begin{array}{l}{[\ldots . .] \text { se a gente não tiver uma padronização eu acho que é o melhor meio da gente }} \\
\text { conseguir um bom material (ENF16). }\end{array}$ \\
\hline 6 & $\begin{array}{l}\text { Creio que auxilia, mas é necessário a implantação de uma comissão composta por } \\
\text { pessoas capacitadas para realizar testes ainda que contando com a ajuda dos } \\
\text { responsáveis de cada setor (MED1). }\end{array}$ \\
\hline 8 & $\begin{array}{l}\text { Porque aí dá uma possibilidade de as pessoas que vão utilizar o material, dentro do } \\
\text { centro cirúrgico ou no setor, possam avaliar se ele atende as necessidades (MED7). }\end{array}$ \\
\hline Fonte: Participantes deste estudo, dados das entrevistas. \\
quando a gente faz a licitação a gente escolhe o material antes de eu gastar [...]
\end{tabular}


Quadro IV - A criação de uma Comissão de Padronização minimiza problemas da qualidade dos materiais hospitalares, Hospital Universitário, Rio de Janeiro, 2015.

\begin{tabular}{|l|l|}
\hline Ordem & Unidades de registro \\
\hline 1 & $\begin{array}{l}\text { Sim (ENF5); Com certeza (ENF4); Acredito sim (ENF7); Sim, eu acho que é essencial } \\
\text { (ENF16); Seria importantíssimo, seria o ideal (ENF17). }\end{array}$ \\
\hline 2 & $\begin{array}{l}\text { Tanto em relação ao material já comprado quanto aos que a comissão nos solicitar } \\
\text { pareceres técnicos (ENF1). }\end{array}$ \\
\hline 3 & $\begin{array}{l}\text { Com certeza! Porque é você seguindo todo um protocolo de uma Comissão avaliando } \\
\text { com certeza você vai ter, você vai adquirir produtos melhores (ENF2). }\end{array}$ \\
\hline 4 & $\begin{array}{l}\text { Eu acredito que sim, que uma comissão de padronização seria eficiente nisso, colocaria } \\
\text { determinadas normas para que fossem adquiridos materiais de boa qualidade (ENF8). }\end{array}$ \\
\hline 5 & $\begin{array}{l}\text { Acredito, desde que a comissão funcione desde que as pessoas estejam na comissão } \\
\text { tenham o compromisso com o trabalho, conhecimento do que está fazendo em fim } \\
\text { (MED2). }\end{array}$ \\
\hline 7 & $\begin{array}{l}\text { Ora se não resolver, eu tenho impressão que ajudaria bastante, porque pelo menos a } \\
\text { gente teria oportunidade de avaliar o material antes da compra (MED3). }\end{array}$ \\
\hline 8 & $\begin{array}{l}\text { Se vai resolver eu não sei, mas que é importante ter é (MED4). } \\
\text { i...] eu não posso dizer que sim, que resolveria, mas, certamente seria um passo } \\
\text { quem fosse utilizar (MED7) }\end{array}$ \\
\hline 9 & $\begin{array}{l}\text { Eu não sei se isso é viável, eu não sei se isso já é feito na verdade [...] eu não tenho } \\
\text { experiência em gestão, eu tenho experiência em ver doente (MED10). }\end{array}$ \\
\hline Fonte: Participantes deste estudo, dados das entrevistas.
\end{tabular}

A análise das Unidades de Registro apresentada nos quadros I,II,III,IV possibilitou emergir as seguintes categorias temáticas: 1) Materiais com mais problemas: intercorrências por falta de critérios de qualidade; 2) Credibilidade sobre solicitação de amostras para o pregão; e 3) Propósitos da comissão de padronização de materiais hospitalares.

\section{Discussão}

Categoria 1: Materiais com mais problemas: intercorrências por falta de critérios de qualidade

Como pode ser observado na tabela I, os participantes deste estudo relatam que os materiais que apresentam mais problemas são: jelcos, luvas, equipos, polifix, cateter venoso e agulhas, além da informação de que nenhum material apresenta problema, como relatos de ENF14, MED4, MED9.

De acordo com estudo realizado em 2012, em instituições hospitalares de todo o território brasileiro, visando identificar as atividades dos enfermeiros gestores de centro de material e esterilização, utilizando-se da ferramenta Google Docs Offline®, a incorporação de tecnologias sofisticadas para aquisição dos materiais pode ajudar no aspecto celeridade, porém, carece de critérios de qualidade dos produtos. Critérios seletivos para dar conta da complexidade das práticas assistenciais hospitalares, que possibilitem realizar 0 monitoramento da qualidade dos produtos, preservando a segurança para o paciente e equipe de saúde [10].

A necessidade de se estabelecer critérios de controle da qualidade dos materiais hospitalares tem preocupado pesquisadores mundo afora. Estudos realizados nos Estados Unidos da América [11], na Inglaterra [12], no Irã [13] e no Canadá [14] confirmam que os avanços tecnológicos da indústria devem ser criteriosamente avaliados antes de utilizados nos procedimentos terapêuticos realizados nas instituições de saúde.

Como constatado nos relatos dos participantes deste estudo, os procedimentos invasivos realizados em unidades de internação carecem de que os materiais neles utilizados sejam de qualidade confiável, sejam testados antes de adquiridos. Em caso de perfuração da luva, a transferência de microrganismos e demais patógenos, é facilitada, aumentando o risco de contaminação do sítio cirúrgico capaz de desencadear infecção hospitalar [15].

Sabe-se que a ocorrência de incidentes que comprometam a segurança do paciente tornou-se um desafio premente para todas as instituições de serviços de saúde, podendo ou não envolver a lesão. Quando ocorrem são denominados eventos adversos [14]. A questão da segurança do paciente tornou-se mais e mais generalizada dentro das instituições de saúde e 
entre os profissionais de saúde no que diz respeito à busca constante pela qualidade dos cuidados prestados e minimizar o número de incidentes evitáveis [16].

As unidades de registro elencadas na tabela II oferecem leitura dos relatos dos participantes, justificando suas avaliações com importante destaque para a falta de qualidade dos materiais, como relatos atribuídos a má qualidade dos cateteres de punção venosa tipo jelco: como relatados pelos participantes ENF3 e ENF8. Destaque também para o sofrimento, estresse e ansiedade [11,12], devido aos procedimentos invasivos que são realizados várias vezes [13] nos clientes em geral, relatados pelo MED3, ou impostos ao recém-nascido, como relatos do participante ENF2.

A temática segurança do paciente encontra-se intrinsecamente relacionada à qualidade nos serviços de saúde e vem sendo amplamente referida e discutida pelos setores prestadores de serviços de saúde, pelas entidades de classe e pelos órgãos governamentais [16-18].

Ausência de critério para avaliação da qualidade dos produtos adquiridos através dos pregões tem causado problemas para a segurança nos hospitais. Uma peculiaridade importante do pregão eletrônico é a chamada inversão das fases de habilitação e julgamento, entendendo-se a primeira como aquela em que o licitante deve comprovar sua capacidade técnica e financeira, além de sua idoneidade fiscal, para fornecer o bem ou serviço que a Administração deseja adquirir. A segunda se refere à apuração efetiva da proposta comercial mais vantajosa para a Administração [19].

Da mesma forma, de acordo com a tabela II, os participantes deste estudo atribuem problema de baixa qualidade dos materiais utilizados para procedimentos de alta complexidade, como cateteres de punção venosa profunda e equipos utilizados para infusão de grandes volumes líquidos, hemotransfusões e diversos medicamentos, como relatados pelos participantes MED1 e MED7.

Entre os riscos intrínsecos na assistência à saúde, a infusão de medicamentos é uma preocupação constante. Avanços tecnológicos da indústria de equipamentos hospitalares viabilizaram a aplicação de medicamentos por via endovenosa através de bombas infusoras. Qualquer erro de programação em uma bomba de infusão pode gerar graves consequências, podendo ser fatal dependendo do perfil do paciente ou da medicação utilizada [20].

Acredita-se que para o estabelecimento e manutenção da segurança do paciente na terapia intravenosa em unidades de tratamento intensivo se faz necessário maior investimento em pesquisas com níveis de evidência mais elevados, uma vez que estas fornecem maior recomendação para prática [21,22].

Em torno de $80 \%$ dos pacientes hospitalizados recebem terapia por infusão. Com o aumento do uso de terapias intravenosas, tornou-se necessário desenvolver dispositivos para infundir drogas com pressão superior à pressão sanguínea e com precisão elevada. Cerca de $30 \%$ dos danos durante a hospitalização estão relacionados a erros de medicação, os quais trazem também sérias consequências econômicas aos hospitais. O custo anual de danos severos a erros de medicação, nos USA, tem sido estimado em torno de U\$ 76,6 bilhões [20].

Os eventos adversos são comumente associados ao erro humano individual, mas devem-se considerar como desencadeadores as condições de trabalho, os aspectos estruturais e a complexidade das atividades desenvolvidas [18].

\section{Categoria 2: Credibilidade sobre solicitação de amostras para o pregão}

Com a regulamentação do pregão eletrônico, como deliberado pelo Decreto no 5.450/2005, as licitações para aquisição de bens e serviços comuns, incluindo as contratações de serviços contínuos no âmbito da União, passaram a ser feitas preferencialmente por esta ferramenta [22]. A ausência de critérios para que o fornecedor ofereça amostras suficientes dos materiais para avaliação pela equipe que os utilizarão, tornou o pregão limitado às compras com entregas mais rápidas e cotadas pelo menor preço, comprometendo princípios de segurança e qualidade de materiais hospitalares, objeto deste estudo.

Alguns participantes deste estudo relataram que acreditam na eficácia da solicitação de amostra para o pregão, pautados nas experiências vivenciadas noutras instituições ou nos setores que atuam no HUGG, como observado na tabela III, nos relatos do ENF3 e ENF5.

Garantias de maior celeridade, desburocratizando as etapas da licitação, com a inversão das fases e entregas mais rápidas [19], não são suficientes para que o pregão eletrônico cumpra exigências básicas de qualidade nos processos de compras de materiais hospitalares. Tampouco, experiências esporádicas de um ou outro membro da equipe 
consolida comportamento crítico sobre a importância da solicitação de amostras, como política institucional, de acordo com o observado nas informações dos participantes deste estudo.

Outros participantes informaram que as amostras oferecidas são insuficientes para testagem, por isso, não acreditam na eficácia da solicitação de amostras, como demonstrado pela tabela III, de acordo com o narrado pelos participantes ENF2 e ENF10.

Quando o fornecedor não oferece amostras suficientes, corre-se risco de graves acidentes com os clientes e membros da equipe. A propósito, define-se por segurança do paciente a redução a um mínimo aceitável do risco de dano desnecessário associado ao cuidado de saúde. O mínimo aceitável refere-se ao conhecimento científico corrente, descobertas disponíveis e ao contexto no qual o cuidado é dispensado [21].

Diante disso, além da criação de uma comissão para solicitação de amostra nos pregões eletrônicos, os participantes deste estudo recomendaram que a mesma seja composta por profissionais capacitados para a tarefa de avaliar a qualidade dos materias que utilizarão nos procedimentos terapeuticos desenvolvidos nos pacientes internados.

Entre os participantes favoráveis à eficácia da solicitação de amostras no pregão, destacou-se o entendimento que a comissão deve ser composta por profissionais capacitados e que sejam envolvidos com a utilização dos materiais, de acordo com o relatado pelo MED1 e MED7, como observado na tabela III.

\section{Categoria 3: Propósitos da comissão de padronização de materiais hospitalares}

Experiências com processos de compras de materiais hospitalares que contemplam critérios para avaliação de amostras são decisivas para que os gestores acreditem que sua implementação é fundamental para o controle da qualidade dos produtos. Vale destacar a importância do preparo profissional para atuação conforme as práticas preconizadas, de modo que estes se tornem capazes de agir corretamente perante as recomendações e assim favoreçam a segurança do paciente [21].

Estudo realizado em hospital de ensino da Cidade de Ribeirão Preto, São Paulo, reitera que a função de compras engloba todas as atividades essenciais associadas à aquisição de materiais, serviços e equipamentos utilizados nos procedimentos terapêuticos realizados na instituição, buscando conciliar requisitos de qualidade, melhor preço e padronização dos itens de maior consumo nas unidades de internação [7].

De acordo com as unidades de registro da tabela IV, os participantes deste estudo relataram acreditar que a criação de uma Comissão de Padronização resolve problemas da qualidade dos materiais hospitalares, como relatos do ENF4, ENF5, ENF7, ENF16 e ENF17. Enquanto houve quem se mostrasse favorável, porém, com reservas, a exemplo do relato do MED4 e MED7.

A inserção e a tentativa de unificação dos conteúdos sobre segurança do paciente ainda é uma proposição recente nas escolas do Brasil e não faz parte dos objetivos escolares. Demonstra a necessidade de uma revisão dos Projetos Pedagógicos, em que se contemple uma abordagem interdisciplinar, bem como transdisciplinar, uma vez que há mudanças contínuas na sociedade contemporânea, e a universidade deve estar à frente dessas discussões [23].

Relevante observar que nas unidades de registro da tabela IV alguns relatos dos participantes destacam que a criação da comissão pode contribuir para melhoria da qualidade do material, como observado nas falas do ENF1 e ENF8.

A propósito do destacado pelos enfermeiros participantes deste estudo, pesquisa realizada em um hospital universitário de médio porte em Belém, Estado do Pará, Brasil, em 2014, sugere que a atuação do enfermeiro na gestão de recursos materiais é uma das maiores conquistas na tomada de decisões gerenciais, o que reforça a sua importância nos aspectos técnicos e administrativos inerentes aos processos de atenção e gestão [24].

Por fim, cabe ressaltar que a administração de instituições hospitalares mantidas com verbas públicas, atualmente, constitui imenso desafio para os gestores, considerando aspectos como baixo financiamento, desequilíbrio entre oferta e demanda, elevados custos e planejamento e políticas públicas ineficientes [7].

O estudo aponta para exposição de pacientes e equipes profissionais causados pela má qualidade dos materiais hospitalares adquiridos através do pregão eletrônico, considerando 
a falta de critérios de avaliação prévia dos produtos, o que poderia ser minimizado com a implementação de uma comissão para testagem dos materiais a serem comprados nesse processo.

A ocorrência de problemas decorrentes do uso de materiais hospitalares de não conformidade causa dor e sofrimento para os clientes internados nos diversos setores do hospital, além de estresse e riscos de acidentes a que ficam expostos os profissionais da equipe terapêutica. Razões que justificam os depoimentos dos participantes deste estudo quando afirmam a necessidade de padronização desses materiais e da sua testagem antes dos processos de compras, realizados através dos pregões eletrônicos.

Diante do avanço tecnológico que os pregões eletrônicos trouxeram para os processos de compras de materiais hospitalares, importante considerar que a celeridade sem critérios de avaliação dos produtos para fins terapêuticos, aumenta a ocorrência da não conformidade, o descarte de vários insumos, o desperdício de recursos financeiros, que bem melhor poderiam ser utilizados em pesquisas acadêmicas da comunidade universitária.

Este estudo não esgota o tema, mas abre horizontes para que outras investidas acadêmicas possam descortinar novas possibilidades na área de administração de compras de materiais hospitalares.

\section{Referências}

1. Faria ER, Ferreira MAM, Santos LM, Silveira SFR. Fatores determinantes na variação dos preços dos produtos contratados por pregão eletrônico. Rev Adm Pública 2010;44(6):1405-28.

2. Oliveira BCSCM, Santos LML. Compras públicas como política para o desenvolvimento sustentável. Rev Adm Pública 2015;49(1):189-206.

3. Bogo PC, Bernardino E, Castilho V, Cruz EDA. The nurse in the management of materials in teaching hospitals. Rev Esc Enferm USP 2015;49(4):632-9.

4. Paschoal MLH, Castilho V. Implementation of computerized material management system at the University of São Paulo University Hospital. Rev Esc Enferm USP 2010;44(4):984-8.

5. Brasil. Decreto o 5.450 , de 31 de maio de 2005. Regulamenta o pregão, na forma eletrônica, para aquisição de bens e serviços comuns, e dá outras providências. Presidência da República. [Internet]. 2005 Mai [cited 2016 Jul 19]; Available from: http://www.planalto.gov.br/ccivil_03/_ato2004-2006/2005/decreto/d5450.htm.

6. Caldas EL, Nonato RS. Compras públicas: estratégia e instrumento para a gestão do desenvolvimento local. Interações (Campo Grande) 2014;15(1):161-72.

7. Duarte NCM, Bitar JPS, Miglioli JP, Pereira MMS, Yodono NBP, Araújo TR, et al. Gestão de compras em um hospital de ensino terciário: um estudo de caso um estudo de caso. Medicina (Ribeirão Preto) 2015;48(1):48-56.

8. Biderman R, Macedo LSV, Monzoni M, Mazon R (Org). Guia de compras públicas sustentáveis: uso do poder de compra do governo para a promoção do desenvolvimento sustentável. 2. ed. Rio de Janeiro: FGV; 2008.

9. Minayo MCS. O desafio do conhecimento: a pesquisa qualitativa em saúde. São Paulo: Hucitec; 2010.

10. Gil RF, Camelo SH, Laus AM. Ana Maria. Nursing tasks in the Material Storage Center of hospital institutions. Texto Contexto Enferm 2013;22(4):927-34.

11. Mannan MS, Reyes-Valdes O, Jain P, Tamim N, Ahammad M. The evolution of process safety: current status and future direction. Annu Rev Chem Biomol Eng 2016;7(7):13562.

12. Davis NF, Quinlan MR, Bhatt NR, Browne C, MacCraith E, Manecksha R, et al. Incidence, cost, complications and clinical outcomes of iatrogenic urethral catheterisation injuries: A prospective multi-institutional study. J Urol 2016;196(5):147377.

13. Asgari H, Esfahani SS, Yaghoubi M, Javadi M, Karimi S. Investigating selected patient safety indicators using medical records data. J Educ Health Promot 2015;4:54.

14. Kemp KA, Santana MJ, Southern DA, McCormack B, Quan H. Association of inpatient hospital experience with patient safety indicators: a cross-sectional, Canadian study. BMJ Open 2016;6(7):e011242. 
15. Oliveira AC, Gama CS. Surgical antisepsis practices and use of surgical gloves as a potential risk factors to intraoperative contamination. Esc Anna Nery Rev Enferm 2016; 20(2): 370-7.

16. Lorenzini E, Santi JAR, Bao ACP. Patient safety: analysis of the incidents notified in a hospital, in south of Brazil. Rev Gaúch Enferm 2014; 35(2):121-7.

17. Tase TH, Lourencao DCA, Bianchini SM, Tronchin DMR. Patient identification in healthcare organizations: an emerging debate. Rev Gaúch Enferm 2013;34(3):196-200.

18. Oliveira RM, Leitão IMTA, Silva LMS, Figueiredo SV, Sampaio RL, Gondim MM. Strategies for promoting patient safety: from the identification of the risks to the evidence-based practices. Esc Anna Nery Rev Enferm 2014;18(1):122-9.

19. Freitas M. Investigação das causas dos problemas de gestão e execução dos contratos de prestação de serviços contínuos, licitados por pregão eletrônico, pela Diretoria de Administração do Campus [Dissertação]. Rio de Janeiro: ENSP-FIOCRUZ; 2011.

20. Holsbach LR, Kliemann Neto FJ, Holsbach N. Utilização do instrumento de identificação de conhecimentos para administração segura de medicamentos com o uso de infusão automática. Rev Bras Eng Bioméd 2013;29(4):353-62.

21. Alves KYA, Costa TD, Barros AG, Lima KIN, Santos VEP. Patient safety in intravenous therapy in the intensive care unit. Rev Pesqui Cuid Fundam 2016;8(1):3714-24.

22. Freitas M, Maldonado JMSV. O pregão eletrônico e as contratações de serviços contínuos. Rev Adm Pública 2013;47(5):1265-81.

23. Bohomol E, Freitas MAO, Cunha ICKO. Ensino da segurança do paciente na graduação em saúde: reflexões sobre saberes e fazeres. Interface Comun Saúde Educ 2016;20(58): 727-41.

24. Silva MJNS, Ribeiro AL. Gestão em centro cirúrgico: identificação de desperdícios. Rev SOBECC 2016;21(2):82-9. 